\title{
Short term production management in the context of cyclic delivery schedules
}

\author{
Z. Bahroun ${ }^{1 \& 2)}$, P. Baptiste ${ }^{2)}$, J.P. Campagne ${ }^{3)}$, M. Moalla ${ }^{\text {l) }}$ \\ 1) Productique et Informatique d'Entreprise \\ Faculté des Sciences de Tunis, Dép. des Sciences de \\ l'Informatique, 1060 Le Belvédère Tunis, Tunisie. \\ $\mathrm{Tel}:(2161) 872600$ \\ Fax : (216 1) 885190 \\ 2) Laboratoire d'Automatique de Besançon \\ 25 Rue Alain Savary, 25000 Besançon, France. \\ $\mathrm{Tel}:$ (33 3) 81407800 \\ Fax : (33 3) 81402809 \\ ${ }^{3)}$ Systèmes Industriels Coopératifs \\ 158 Cours Fauriel, 42023 Saint-Etienne Cedex 2, France. \\ Tel : (33 4) 77420123 \\ Fax : (33 4) 77420000
}

\begin{abstract}
This paper considers a factory which produce $\mathrm{n}$ items on a single facility and which is confronted to a cyclic demand: each item is ordered relatively to a cyclic delivery schedule with a mean quantity for each delivery. This kind of " contractorder " is found very frequently, especially in the big distribution and the automotive sectors. So, the long-term information becomes the due date and the exact quantity that will be delivered is only known in the short term.

This work aims at defining a new production management model which considers these new commercial paradigms.

In a long-medium term we have calculated and generated cyclic production schedules. In a short term we present capacities adjustments approaches and safety stocks determination.
\end{abstract}

Key words

production management, just in time, cyclic scheduling, cyclic delivery schedule. 


\section{INTRODUCTION}

The supply chain integration and the emergence of new paradigms issued from the just in time philosophy have called into question the existing production management methods.

Companies slowly moved from the classical order between a customer and a supplier with a quantity and a delay to the notion of a " contract-order » in which the supplier is linked with the customer through a cyclic delivery schedule with a forecasted quantity for each delivery. This kind of order is found very frequently, especially in the big distribution and the automotive sectors. So, the long-term information becomes the due date and the exact quantity, which will be delivered, is only known in the short term.

The work presented in this paper aims at defining a new production management model taking into consideration these new paradigms.

A large bibliographical study has been carried out and concerns mainly the cyclic scheduling. The study shows as evidence that the global problem is so complex that it is necessary to split it in several steps. In our work, we propose a decomposition of the problem in three steps:

1. Determination of the global production cycle and the production frequency of the different items into the cycle (based on mean demands).

2. Determination of a repetitive sequencement that takes into account the obtained results in the first step and which is optimized relatively to specific criteria.

3. Modelization of the capacity problems (arbitration between over-time and subcontracting) and determination of the final batch size responding to shortterm events. The third step includes also the safety stocks determination.

This subdivision corresponds also to a temporal decomposition. The first and the second step correspond to a long-medium term level and the third one corresponds to a short-term level.

\section{PRODUCTION FREQUENCIES DETERMINATION}

In a previous paper (Bahroun, 98c), we have focused on the first sub-problem. We have considered a factory which produces $\mathrm{n}$ items on a single facility and which is confronted to a cyclic delivery schedule (several independent deliveries of the same product in the cycle, for instance, we must deliver the finished product 1 , 1000 units each Monday, 2000 each Tuesday and 5000 each Friday, the delivery cycle in this case is the week). We have modelized a total cost function, which regroups set-up, ordering and inventory carrying costs (raw material and finished products). We have then developed heuristics to determinate a cycle time, a production frequency for each item in the cycle and a batch size. Those parameters must minimize the model total cost function. 
The total cost function T.C. can be evaluated as:

$$
\begin{aligned}
& T C=\sum_{j=1}^{n}\left[\frac{1}{y_{j}} *\left(C r_{j}+\sum_{k=1}^{v} z_{j, k} * C l_{k}\right)\right] \frac{1}{T}+ \\
& \sum_{j=1}^{n}\left[\frac{1}{2}\left(1-\frac{X_{j}}{L_{j} P_{j}}\right) S f_{j}+\frac{X_{j}}{2 L_{j} P_{j}}\left[\sum_{k=1}^{v} r_{j, k} S p_{k}\right]\right] \frac{X_{j} y_{j}}{L_{j}} T+\sum_{j=1}^{n} \frac{1}{2}\left(X_{j}-2 B_{j}\right) S f_{j}
\end{aligned}
$$

Where: ${ }^{B}=\frac{\left(\sum_{i=1}^{w-1} x_{i j} \sum_{v=i+1}^{w j} l_{v j}\right)}{L_{j}}$

$\mathrm{n}=$ number of finished products (F.P.)

$Q_{j}=$ batch size of the F.P. $j$

$\mathrm{T}_{\mathrm{j}}=$ time interval between two productions of the F.P. $\mathrm{j}$

$\mathrm{T}=$ production cycle duration

$f_{j}=$ production number of the F.P.j in a cycle $T$

$\mathrm{y}_{\mathrm{j}}=[\mathrm{Tj} / \mathrm{T}]$

$\mathrm{R}_{\mathrm{j}}=$ set-up time of the F.P. $\mathrm{j}$

$\mathrm{P}_{\mathrm{j}}=$ production rate of the F.P. $\mathrm{j}$

$\mathrm{v}=$ number of raw material (R.M.) references

$\mathrm{z}_{\mathrm{j}, \mathrm{k}}=$ boolean indicating if the F.P.j use the raw material $\mathrm{k}$

$\mathrm{r}_{\mathrm{j}, \mathrm{k}}=$ quantity of raw material $\mathrm{k}$ used by the F.P. $\mathrm{j}$

$\mathrm{Sf}_{\mathrm{j}}=$ holding cost of the F.P. $\mathrm{j}$ by unit product and unit time

$\mathrm{Sp}_{\mathrm{k}}=$ holding cost of the R.M. $\mathrm{k}$ by unit product and unit time

$\mathrm{Cr}_{\mathrm{j}}=$ manufacturing set-up cost of the F.P.j

$\mathrm{Cl}_{\mathrm{k}}=$ ordering cost of the R.M. $\mathrm{k}$

$\mathrm{w}_{\mathrm{j}}=$ delivery number of the F.P.j in the cyclic delivery schedule

$\mathrm{x}_{\mathrm{uj}}=$ the $\mathrm{u}^{\text {th }}$ delivery quantity of the F.P.j in the cyclic delivery schedule

$1_{\mathrm{uj}}=$ time interval between the $\mathrm{u}^{\text {th }}$ delivery and the $(\mathrm{u}-1)^{\text {th }}$ one for the F.P.j

$X_{j}$ represents the total delivery quantity by delivery cycle and $L_{j}$ the total duration of the delivery cycle

$$
X_{j}=\sum_{u=1}^{w_{j}} x_{u j} \quad L_{j}=\sum_{u=1}^{w_{j}} l_{u j}
$$

The optimal solution consists at :

Calculating for each item the optimal production cycle $\mathrm{T}_{j}{ }^{*}$ by considering each time only one variable $T_{j}$ (which is equal to $y_{j} T$ ) and deriving the T.C. function relatively to $T_{j}$.

Taking as global cycle $T$ the LCM of all the $T_{j}$.

The problem is that generally we obtain a big global cycle. An important cycle leads to the loss of the benefits which can be obtained with a cyclic production. So, we have decided to try to find out a smaller cycle with a small difference in the cost in comparison of the optimal cycle.

The first approach consists at calculating the total cost of all the possible cycles until a fixed value and testing at each time the difference with the optimal 
solution. We choose after that the best cycle from those inferior to a fixed value. The experiments show that the difference is generally inferior to 2 per cent.

The second approach consists at using an heuristic program. This heuristic determines the production cycle and the different $y_{j}$ (the production frequency of each item $\mathrm{j}$ in the global cycle T). It is mainly composed of three steps:

Step 1: -We calculate for each item the optimal production cycle $\mathrm{T}_{j}{ }^{*}$ by considering each time only one variable $T_{j}$ (which is equal to $\mathrm{y}_{j} \mathrm{~T}$ ) and deriving the T.C. function relatively to $T_{j}$.

-We fix arbitrarily all the $y_{j}$ to 1 in the T.C. function.

Step 2: The T.C. function becomes mono-variable (T), we calculate the optimal cycle $T^{*}$ by deriving the T.C. function relatively to $T$.

Step 3: We calculate the new values of $y_{j}{ }^{*}\left(\right.$ equal to $\left.T_{j}{ }^{*} / T^{*}\right)$. If the rounds of $y_{j}$ differ from those found in the precedent iteration we repeat the steps 2 and 3.

The second step consists on calculating $\mathrm{T}^{*}$ with the new values of $\mathrm{y}_{\mathrm{j}}{ }^{*}$. We test both the inferior and the superior round of $y_{j}{ }^{*}$.

Step 4: We calculate then the batch sizes with this formula:

$\forall \mathrm{j} Q_{j}=T_{\text {opt }} \frac{x_{j}}{l_{j}} y_{j}$ final

$\left(y_{j}{ }^{\text {final }}\right.$ is the last value of $y_{j}$ and $T_{\text {opt }}$ is the last $T^{*}$ calculated with the $y_{j}^{\text {final }}$ ).

We calculate after that $\underline{u}$ the $\mathbf{L C M}$ of the $\mathbf{y}_{\mathbf{i}}^{\text {final }}$. Then, we determine the production

frequency $\mathrm{f}_{\mathrm{j}}:{ }^{\forall} f_{j}{ }^{\text {final }}=\frac{u}{y_{j} \text { final }}$

In a global cycle that is equal to $u$ times the base cycle $T_{\text {opt }}$, we will produce $f_{j}$ times the product $\mathrm{j}$ :

$$
T_{\text {global }}=T_{\text {opt }} * u
$$

The experiments shows also that the difference in costs of the cycle obtained with this heuristic and the optimal one is not important (generally inferior to 4 per cent).

\section{SEQUENCE AND PHASE DETERMINATION}

For the second problem of our global philosophy (Bahroun, 1998c). We have determinate from the production frequencies, the best production sequence and phase. This determination is based on the Pinto-Mabert sequence generation and on a linear program to determinate for each sequence the best phase in terms of minimizing the carrying costs and respecting in the same time the deliveries.

In this paper, we focus on the second problem of our global philosophy. Our goal is to determinate from the production frequencies, the best production sequence and phase. This determination is based on the Pinto-Mabert sequence generation (Kim, Mabert and Pinto, 1993, 1995) and on a linear program to determinate for each sequence the best phase. 
The initial sequence ( Initial Sequence Generation Algorithm ISGA) is generated in three steps:

The step 1 consists on calculating the total number of batches (b) in a repetitive cycle, and the number of cells $\left(l_{k}\right)$ in the $k^{\text {th }}$ sub-cycle such that:

$$
b=\sum_{j} f_{j} \text { and } l_{\mathrm{k}}=[\mathrm{b} / \mathrm{u}]^{+}=l^{\forall} \mathrm{k} \text {. }
$$

The second step consists on sorting the items in the ascending order of $f_{j}$. If there are ties among the $f_{j}$ 's we sort in the ascending order of the processing time per batch.

The third step consists on assigning the ranked, sorted items to cell $\mathrm{SC}_{\mathrm{ik}}\left(\mathrm{i}^{\text {th }}\right.$ cell in the $\mathrm{k}^{\text {th }}$ sub-cycle; $\left.\mathrm{i}=1, \ldots, l_{\mathrm{k}} ; \mathrm{k}=1, \ldots, \mathrm{u}\right)$ until all items are assigned, using:

Rule 1: Cell order $\left(\mathrm{O}\left(\mathrm{SC}_{\mathrm{ik}}\right)\right)$ arrangement

$\mathrm{O}\left(\mathrm{SC}_{\mathrm{ik}}\right)=$ ascending order of $\mathrm{k} / \mathrm{i}=2 \mathrm{p}-1$, descending order of $\left.\mathrm{k} / \mathrm{i}=2 \mathrm{p}\right\}$, where $\mathrm{O}\left(\mathrm{SC}_{\mathrm{ik}}\right)=$ cell number by which an item is assigned; $\mathrm{p}^{\in} \mathrm{N}^{+}$.

Rule 2 : Sub-cycle skipping interval $\left(\mathrm{SI}_{\mathrm{j}}\right)$ determination

$$
\mathrm{SI}_{\mathrm{j}}=\left(\mathrm{u} / \mathrm{f}_{\mathrm{j}}\right)-1 \forall \mathrm{j}
$$

We generate after that the dominant sequences by swapping the products having the same frequency in the initial sequence.

After generating the dominant sequences, we must found the best phase of each sequence (the phasing consists on placing the repetitive sequence in the time) using a linear program. The best phase is the one, which minimize the necessary initial stocks that ensure that the stock of all the products remain superior to zero at any time.

For each sequence and phase we must calculate the necessary stocks, we will use for this a linear program.

We will cut the time in $\mathrm{h}$ sub-periods (the sub-period may be the hour) and we will define this program:

$\delta_{i, t}=1$ if the product $i$ is in production in the sub-period $t$ ( 0 if not $)$

$\psi_{t, i}=1$ if the product $i$ will be delivered in the sub-period $t$ ( 0 if not)

So, the stock of the product $i$ at the end of the sub-period $t$ can be calculated with this formula:



The necessary initial stocks for the sequence and the phase can be given by 


$$
\operatorname{Min}\left(\sum_{i=1}^{n} \sum_{t=1}^{T} S_{t, i}\right)
$$

with $\quad \mathrm{S}_{\mathrm{t}, \mathrm{i}}>=0^{\forall} \mathrm{t}$

\section{CAPACITY ADJUSTMENT AND SAFETY STOCKS DETERMINATION}

In this paper, we focus on the last sub-problem, the short term production management.

The cyclic schedule determined in the precedent steps is based on mean demands. The definitive quantities of the orders are known only few days before the delivery. So, we propose an approach to adjust the necessary capacity considering the definitive order quantities. We have defined for this, a linear program which minimize the difference between the starting date of the order and the latest starting date of this order considering the quantities and the dates of the deliveries covered by this order:

$$
\operatorname{Min} \sum_{i=1}^{K}\left(d_{i}-\operatorname{Min}\left(\left(\lambda_{u i}-\sum_{v=1}^{u} \frac{y_{u i}}{R_{i}}\right)\right)\right)
$$

where:

$\mathrm{x}_{\mathrm{i}}=$ the supplementary capacity associated to the order $\mathrm{i}$.

$\mathrm{d}_{\mathrm{i}}=$ the starting date of the order $\mathrm{i}$.

$e_{i}=$ the ending date of the order $i$

$\mathrm{q}_{\mathrm{i}}=$ the quantity of the order $\mathrm{i}$.

$\mathrm{X}=$ the total supplementary capacity.

$\mathrm{K}=$ the total number of the orders.

$R_{i}=$ production rate of the finished product $j$.

$y_{u i}=$ the $u^{\text {th }}$ delivery quantity covered by the order $i$ in the cyclic delivery schedule.

$\lambda_{\text {ui }}=$ date of the $u^{\text {th }}$ delivery covered by the order $i$.

$U_{i}=$ the number of deliveries covered by the order $i$.

$\mathrm{s}, \mathrm{r}=$ variables fixed by the user to limit the supplementary capacity allocated to an order in comparison of his own production rate.

With these constraints:

$$
\begin{gathered}
\mathrm{d}_{1}=0 \\
\forall \mathrm{i}>1 \quad d_{i}=d_{i-1}+\left(\frac{q_{i-1}}{R_{i-1}}-x_{i-1}\right)
\end{gathered}
$$


$\forall_{\mathrm{i}} \quad d_{i} \leq \operatorname{Min}\left(\left(\lambda_{u i}^{U_{i}}-\sum_{v=1}^{u} \frac{y_{u i}}{R_{i}}\right)\right)$

$\forall_{\mathrm{i}} \quad s \frac{q_{i}}{R_{i}} \leq x_{i} \leq r \frac{q_{i}}{R_{i}}$

The originality of this approach is that we allocate a supplementary capacity (negative or positive) to an order and no to a period.

The second problem which must be treated is the safety stocks determination. We have for this also developed an approach for fixing safety stocks. In fact, the safety stocks in our case will cover the uncertainty of the starting date of the different orders. So, each order will produce a safety stock to cover the uncertainty of the starting date of the next order and will consume the safety stock created by the precedent order for covering his uncertainty of his starting date.

The approach consists at estimating the variance of the uncertainty of the starting date of each order of the sequence. Then we can fix a safety stock for each order which can cover two variances.

\section{CONCLUSION}

We have defined in this paper a new management model taking into consideration the cyclic delivery context. We have first of all calculated from a delivery frequency and a mean demand for each delivery, production frequencies and batch sizes for each product. We have used for this three approaches. The first one gives us an optimal solution in cost but with a big cycle. The second one gives us a better solution in terms of the lengthening of the cycle but with a slight difference in the cost in comparison with the optimal solution. The third approach is an heuristic which gives us directly a good solution in terms of cost and lengthening of the cycle. So, by using these three approaches we can choose the best cycle in terms of cost and lengthening of the cycle.

We have after that generated the best sequence and phase of our cyclic production planning. We have used and modified the Pinto-Mabert approach to generate the dominant sequences. We developed then a linear program which find out the best sequence and phase.

We have then studied the short term management. We have proposed in this context approaches to adjust capacities and calculate safety stocks. A linear program has been developed to allocate a supplementary capacity (negative or 
positive) to an order. The safety stocks calculation is based on estimating the variance of the uncertainty of the starting date of each order of the sequence.

This work can be easily extended to a multi-stage and multi-ressources production system. We must only consider that, from stage to stage, we have a suppliercustomer relationship.

We can also the study the long-term management which consists for example to decide if we take a new contract or not and with which conditions.

\section{MAIN REFERENCES:}

Bahroun, Z., (1996). Conception et développement d'une nouvelle approche de planification des charges à capacité finie pour un flow-shop généralisé, Mémoire de D.E.A. Faculté des Sciences de Tunis, CFDI FST-ENSI, Tunisie.

Bahorun, Z., Campagne, J.P. and Moalla, M. (1997). The overlapping production planning : A new approach of a limited capacity management. In : IEPM'97 Industrial Engineering and Production Mangement, France, 20-24 October 1997.

Bahroun, Z., Baptiste, P., Campagne, J.P. and Moalla, M. (1998 a). A production management model operating under a cyclic delivery schedule. In : CESA'98 International Conference, Tunisia, 1-4 April 1998.

Bahroun, Z., Baptiste, P., Campagne, J.P. and Moalla, M. (1998 b). Production planning and scheduling in the context of cyclic delivery schedules. In : ICCIE'98 International Conference on Computers and Industrial Engineering, London, 9-11 September 1998.

Bahroun, Z., Campagne, J.P. and Moalla, M. (1998 c). The overlapping production planning : A new approach of a limited capacity management. Paper submitted and accepted in the I. J. Prod. Econ.

Bahroun, Z., Baptiste, P., Campagne, J.P. and Moalla, M. (1999). Extension of the overlapping production planning and application for the cyclic delivery context. In : IEPM'99 Industrial Engineering and Production Mangement, Scotland, 12-15 July 1999.

Golhar D-Y. and Sarker B-R. (1993). Economic manufacturing quantity in a justin-time delivery system. I.J. Prod. Research, 30, 961-972.

HALL R-W. (1988). Cyclic scheduling for improvement. I.J. Prod. Research, 26, 457-472.

Hill M. R. (1994). Optimizing a production system with a fixed delivery schedule. J. Oper. Research Society, 47, 954-960.

Kim D., Mabert V-A and PINTO P-A. (1993). Integrative cycle scheduling approach for a capacitated flexible assembly system. Decision Science, 24, 126-147. 
Kim D. and Mabert A. (1995). Integrative versus separate cycle scheduling heuristics for capacitated discrete lot sizing and sequencing problems. I. J. Prod. Research, 33, 2007-2021.

Loerch A. G. and Muckstadt J. A. (1994). An approach to production planning and scheduling in cyclically scheduled manufacturing systems. I. J. Prod. Research, 32, 851-871.

Nori V. S. and Sarker B. R. (1996). Cyclic scheduling for a multi-product, single facility production system operating under a just-in-time delivery policy. $J$. Oper. Research Society, 47, 930-935.

Sarker B-R. and Parija G-R. (1994). An optimal batch size for a production system operating under a fixed-quantity, periodic delivery policy.", J. Oper. Research Society, 45, 891-900.

\section{BIOGRAPHY}

Zied Bahroun is an industrial engineer since 1994. He prepared and obtained after that a master in 1996. He's preparing since 1996 a $\mathrm{PhD}$ thesis and he's assistant in the Faculty of Sciences of Tunis (Tunisia). His $\mathrm{PhD}$ thesis is untitled "contributions to the management of cyclic production systems". 